\title{
Duplicity Among Solar-Type Stars
}

\author{
HELMUT A. ABT \& DARYL W. WILLMARTH \\ Kitt Peak National Observatory, Box 26732, Tucson, AZ 85726, USA
}

\begin{abstract}
From a new study of 113 F8-G1 IV or V primaries in an apparentmagnitude limited sample, measured with a CCD for about 20 radial velocities of accuracy $0.2-0.5 \mathrm{~km} \mathrm{~s}^{-1}$ each, we collected data for 28 binary orbits and 39 visual systems. We again found a flat distribution (or slightly decreasing toward lower masses) of secondary masses, unlike the Salpeter distribution for B2-B5 stars. But we find that the different distributions for these field stars and for open clusters of various ages can all be explained by a capture mechanism of binary formation.
\end{abstract}

In 1976, Abt \& Levy published a search for binaries among 135 F3-G2 IV and $\mathrm{V}$ stars and found the following results:

1. The frequency of binaries is considerably higher than $50 \%$ so that double stars are more common than single stars.

2. The distribution of periods shows a single maximum over 10 orders of magnitude with a good overlap of binaries found by different methods (spectroscopic, visual, etc. )

3. The widely-spaced binaries (mostly CPM pairs) show a distribution of secondary masses similar to the van Rhijn distribution (see Figure 1). It was suggested that those binaries were formed by capture.

4. For binaries with periods less than 100 years, the frequencies of various secondary masses show a fiat distribution or one decreasing toward lower masses. Those binaries were attributed to formation by bifurcation because that process favors pairs of equal masses.

Later it was shown (e.g., Durisen \& Tohline 1985) that under the assumption of compressible viscous gases, contracting rotating ellipsoids do not produce double stars by bifurcation, but rather disks and rings. We may learn more about this work during this meeting. But we must remember that whatever way binaries are formed, that process must apply to the majority of all stars.

More recently Abt et al. (1990) studied B2-B5 main-sequence stars and again found that for widely-spaced pairs, the van Rhijn distribution, or better yet for early-type stars, the Salpeter function represents the secondaries (see Figure 2). However it also represents the closely-spaced binaries. Why is it that the closely-spaced B-type binaries show a different distribution in secondary masses than for solar-type stars? We decided to check the original photographic study of solar-type stars, using a CCD and cross-correlation analysis and to avoid the early $\mathrm{F}$-type stars where rotational broadening can cause inaccuracies.

Meanwhile the Geneva group (Duquennoy et al. 1991; Duquennoy \& Mayor 1991; Mazeh et al. 1992) are completing a huge study based on observations taken during 13 years of 280 nearby F0-G9 IV and V stars, based on CORAVEL velocities having an accuracy of $0.2-0.3 \mathrm{~km} \mathrm{~s}^{-1}$. Some of their results are: 


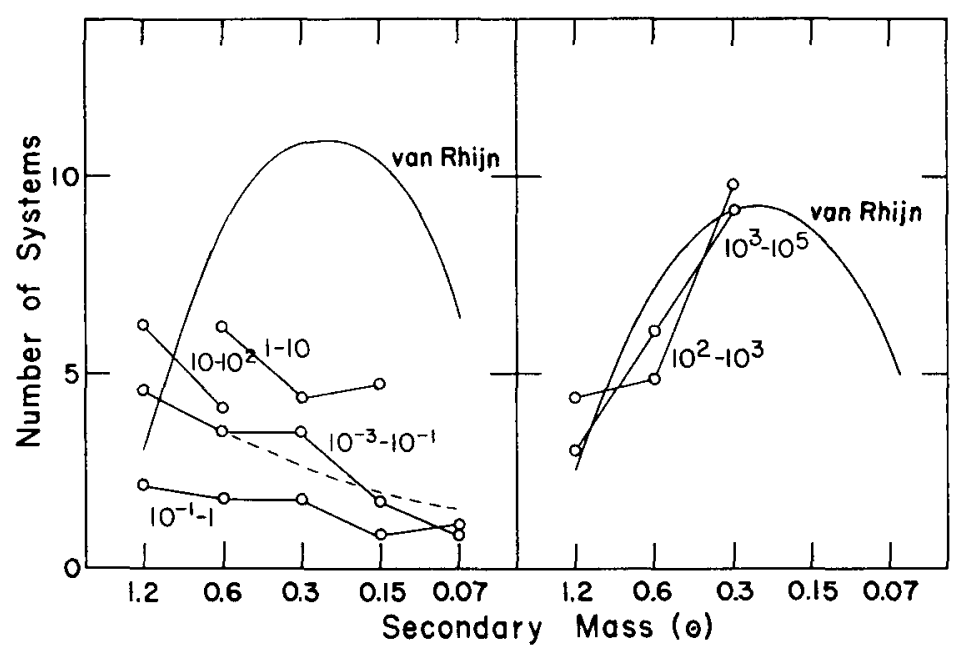

FIGURE 1. From Abt \& Levy (1976) the frequencies of various secondary masses of F3-G2 IV or V primaries are shown for the short-period binaries (left) and long-period binaries (right). The periods are indicated in years.

1. They confirm the high frequency of doubles.

2. They confirm and illustrate very well the single maximum in the period distribution. This single maximum may imply, but does not demand, a single formation mechanism for binaries.

3. They confirm that for shorter periods, the distribution of secondary masses is flat or slightly decreasing toward lower masses.

But, in 1986 we were not aware of the Geneva program and started our own study of solar-type stars when our CCDs became available in our coudé spectrograph, which is fed by light from a $90-\mathrm{cm}$ "coudé feed" telescope. We used in 1986-1990 an 812×812-pixel CCD chip, a dispersion of $15 \mathrm{Amm}^{-1}$, and a 25 -micron pixel size or $0.4 \AA$ resolution. There were initial problems with wavelength shifts as the liquid nitrogen evaporated during the night, decisions about using a normal slit vs. fiber feed, and appropriate filters for the TV guider. As a result of improvements made the initial accuracies of $0.5-0.7 \mathrm{~km} \mathrm{~s}^{-1}$ gradually reduced to $0.2-0.3 \mathrm{~km} \mathrm{~s}^{-1}$. In fact, re-reduction of all the observations are still being made so the following results are based on preliminary data subject to minor changes.

We found or confirmed orbital elements for 28 spectroscopic binaries and collected data on 39 primaries having visual secondaries with orbital elements or that are speckle, occultation, or CPM pairs. After deletions appropriate for a magnitude-limited sample and dropping the high-velocity stars, we find that there are 0.54 observed secondaries for each primary.

An interesting side issue is circularization in short-period binaries. Our data show that all systems with periods less than 8 days have eccentricities less 


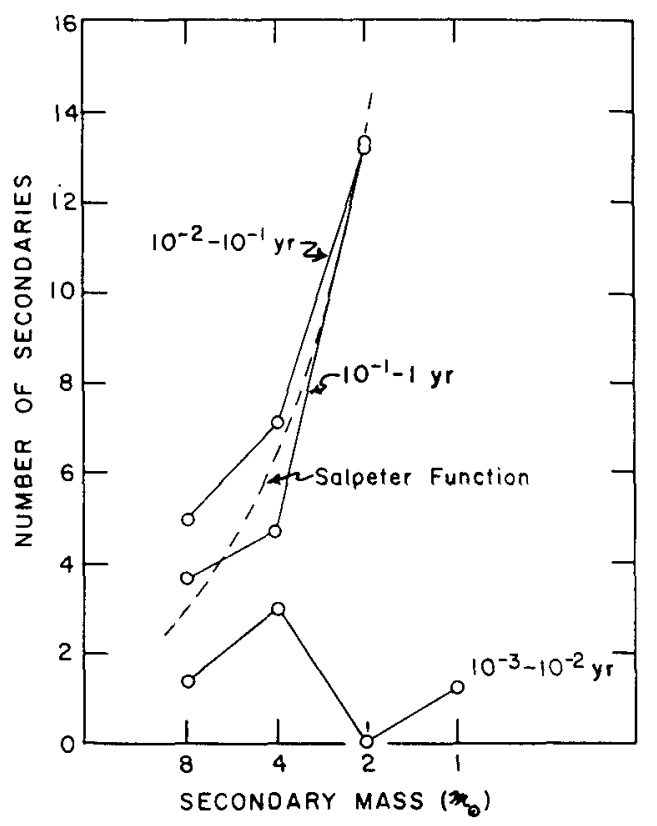

FIGURE 2. From Abt et al. (1990), the frequencies of various secondary masses of B2-B5 IV or V primaries are shown, compared with the Salpeter function. The shortestperiod group (less than 3.6 days) are probably interacting systems with modified mass ratios.

than 0.02 (see Figure 3). The eccentric system with the shortest period is the 9.6-day binary HR $5304=12 \mathrm{~d}$ Boo $=$ HD 123999. Our velocity curve, shown in Figure 4 , is convincingly eccentric at $\mathrm{e}=\mathbf{0 . 2 0}$ and agrees well with Harper's orbital elements in 1914. This is a slightly shorter period than that found by the Geneva group, namely 13.2 days for HD 17433 as the first eccentric system.

For comparison we can look at the results for the Am stars (Abt \& Levy $1985)$ where the cutoff is at 4.8 days. Among the B2-B5 IV and V stars there is no cutoff. But one trouble with interpreting these results in terms of tidal interactions is in trying to assign ages for these field stars.

Returning to the main issue, we computed the secondary mass distributions by computing the mass functions for various period ranges, secondary masses, and inclinations. Then we matched those against the observed mass functions, knowing that the frequencies of various inclinations should vary as the cosines of the inclinations. This then tells us the numbers of secondaries of various masses and allows us to compute which inclinations are unobservable. This method has the same assumptions as that used by the Geneva group, uses a different mathematical fitting procedure, but Halbwachs (1987) has shown that it gives the same results.

The results are given in Figure 5 for periods from a fraction of a day to 100 years. All show a flat or slightly decreasing frequency toward lower masses, 


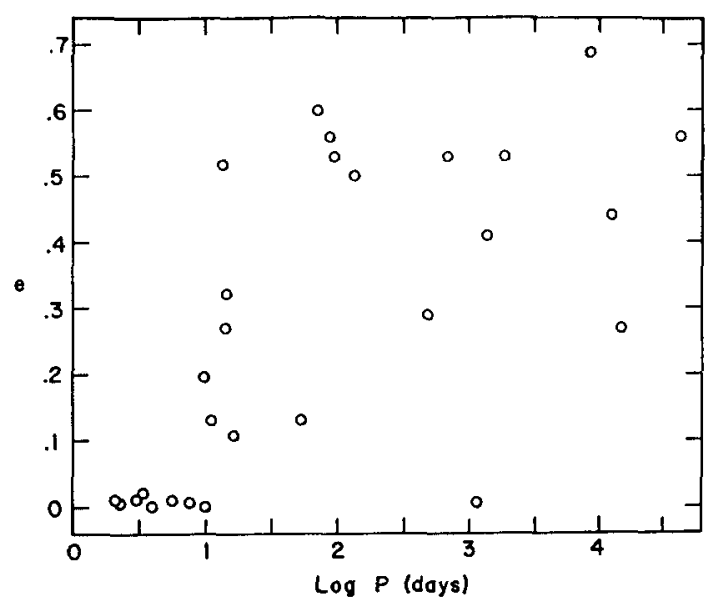

FIGURE 3. The orbital eccentricities of binaries with F8-G1 IV or V primaries are shown.

again confirming the Abt-Levy paper and the Mazeh et al. preprint.

So again we have this difference between the short-period binaries among the solar and B stars, but now we cannot simply attribute the difference to different formation mechanisms, like bifurcation and capture, because bifurcation does not work.

TABLE 1. Histories of 19 Aarseth-Hills Binaries

\begin{tabular}{|c|cccc|}
\hline Time & \multicolumn{4}{|c|}{$\mathrm{q}=\mathrm{M} 2 / \mathrm{M} 1$} \\
(free-fall times) & 1.0 & 0.5 & 0.25 & 0.125 \\
\hline$<0.4$ & 0 & 2 & 4 & 2 \\
$0.4-0.8$ & 2 & 4 & 0 & 0 \\
$>0.8$ & 3 & 1 & 0 & 1 \\
\hline
\end{tabular}

However there are interesting results from people who do n-body simulations of mass points in hypothetical clusters. One study was done by Aarseth \& Hills (1972), who studied a cluster having subclustering, like an association. They followed the formation and disruption of various pairs. They found, as one would expect, that the most massive stars initially pair with low-mass companions because the low-mass stars are so plentiful. This gives a van Rhijn or Salpeter distribution. But, in subsequent three-body encounters the most massive stars gradually shed the low-mass companions in favor of high-mass companions, leading to a flat secondary distribution or one decreasing toward smaller masses. This tells us that in young clusters we should expect the secondaries to follow the Salpeter distribution but in older clusters they should have a flat distribution. 


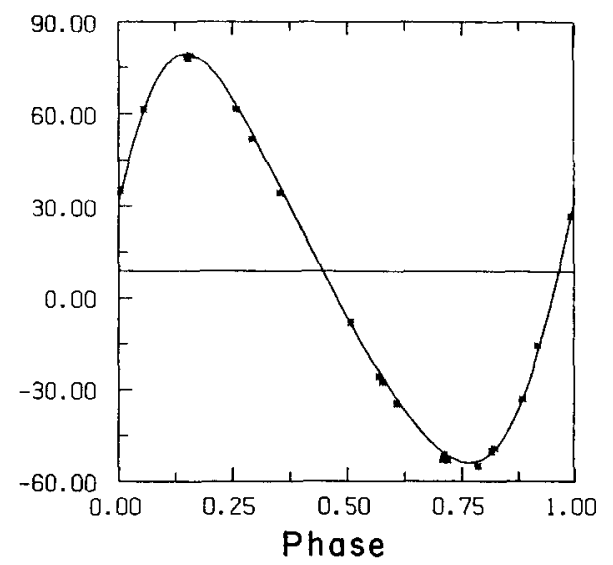

FIGURE 4. The radial-velocity (in $\mathrm{km} \mathrm{s}^{-1}$ ) curve for the primary of HR $5304=$ $12 \mathrm{~d}$ Boo $=$ HD 123999 is shown. The period is $9.6051 \pm 0.0003$ days and the eccentricity is $0.196 \pm 0.004$.

These theoretical results are given in Table 1 that shows what happened to 19 binaries as a function of time measured in free-fall times. Note the van Rhijn-like distribution holds for times much shorter than the free-fall time, but a flat or decreasing distribution holds for times comparable to the free-fall time.

We can compare these expectations with results published in the last two years of observations in three clusters, shown in Table 2. The Orion Nebula cluster (Abt et al. 1991) has an age of one million years and has small secondary masses, even in the cases of two SB2 binaries. The alpha Persei cluster (Morrell \& Abt 1992) with an age of about 10 million years also has only low-mass secondaries, including that of one SB2 system. But IC 4665 (Morrell \& Abt 1991) at 50 million years has two binaries (both SB2s) with mass ratios of 0.97 and 0.88. It is difficult to estimate the free-fall times for these three clusters because we do not know the total masses of these clusters. But the evolutionary changes are very similar to those expected.

TABLE 2. Cluster Results

\begin{tabular}{|l|cc|ccc|}
\hline Cluster & \multicolumn{3}{|c|}{ Ages } & \multicolumn{3}{c|}{$\mathrm{q}$} \\
& $\left(10^{6} \mathrm{yr}\right)$ & (free-fall) & 1.0 & 0.5 & 0.25 \\
\hline Orion Neb. & 1 & $<1$ & 0 & 1 & 3 \\
$\alpha$ Per & 10 & 1 & 0 & 1 & 3 \\
IC 4665 & 50 & 5 & 2 & 1 & 1 \\
\hline
\end{tabular}

The field B2-B5 and solar-type stars also fit this evolutionary pattern. The reason why the widely-spaced binaries, such as CPM pairs, fit the van Rhijn or Salpeter distribution regardless of age is because they are loosely-paired systems 


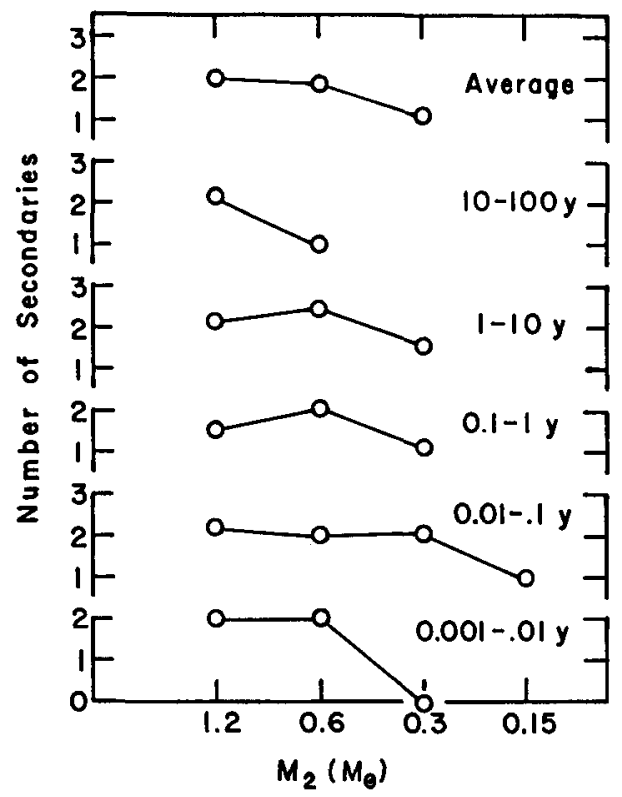

FIGURE 5. For the F8-G1 IV or V primaries, the frequencies of various secondary masses are shown for various period intervals.

that have not gone through a sequence of three-body interactions that generally result in close pairs of nearly equal masses. And it does not matter whether they complete star formation with a companion or not because most initial binaries are disrupted.

Therefore we find that young and old binaries in clusters and in the field can all be understood in terms of binary formation by capture alone.

\section{REFERENCES}

Aarseth, S.L. \& Hills, J.G. 1972, $A \& A, 21,255$

Abt, H.A., Gomex, A. E., \& Levy, S. G. 1990, ApJS, 74, 551

Abt, H.A. \& Levy, S.G. 1976, ApJS, 30, 273

Abt, H.A. \& Levy, S.G. 1985, ApJS, 59, 229

Abt, H.A., Wang, R., \& Cardona, I. 1991, ApJ, 367, 155

Duquennoy, A. \& Mayor, M. 1991, $A \& A, 248,485$

Duquennoy, A., Mayor, M., \& Halbwachs, J.L. 1991, $A \& A, 88,281$

Durisen, R.H. \& Tohline, J.E. 1985 in Protostars and Planets II, ed. D.C. Black and M.S. Mathews, (Tucson: Univ. Arizona Press), p. 534

Halbwachs, J.L. 1987, $A \& A, 183,234$

Harper, W.E. 1914, Pub. Dom. Obs., 1, 303

Maseh, T., Goldberg, D., Duquennoy, A., \& Mayor, A. 1992, ApJ, submitted

Morrell, N. \& Abt, H.A. 1991, ApJ, 378, 157

Morrell, N. \& Abt, H.A. 1992, ApJ, in press 


\section{DISCUSSION}

POVEDA: It is very difficult to make a significant number of wide binaries $(\mathrm{P}>100 \mathrm{yr})$ by capture; the Salpeter mass spectrum is easier to understand in terms of independent condensations in a bound cloud. Years ago, we made N-body computations for small clusters which showed that by far the binaries formed when composed by the two most massive stars in the cluster.

ABT: I rely on the Aarseth \& Hills simulations that assumed realistic parameters and showed in a sequence of binary formations and disruptions that changes in binary characteristics from a secondary Salpeter distribution to the flat distribution found by us, Mazeh and Goldberg, and the Geneva group. In addition, the presence of gas and dust and disks would facilitate these processes.

MATHIEU: You also find a flat secondary mass spectrum for field solar-mass stars. Presuming that these stars were never in clusters where they were the maximum mass stars, how would your mechanism explain such a secondary mass distribution?

ABT: This mechanism of repeated captures and disruptions works only in clusters, and Aarseth \& Hills accelerated the mechanism by assuming dense subclusters in a diffuse association.

MAYOR: During the tidal circularization process, not only the eccentricity diminishes but also the period. So we can observe non-circular orbits at any short period in the course of circularization. The longest period with a circular orbit is much more difficult to determine. In this regard, I am surprised to see that $P_{\max }(\mathrm{e}=0)$ is approximately 10 days for $\mathrm{Am}$ stars as is also the case for $\mathrm{G}$ dwarfs.

MATHIEU: We have a poster paper (Matthews \& Mathieu) showing the eccentricity distribution of A stars, based on binaries in the Batten catalog. Remarkably, circular orbits are found at periods approaching 10 days, just as found among solar-mass binaries in the field. Indeed, two of the longest period circular orbits are double-lined systems, so both components are definitively early spectral types. These circular orbits are at periods much longer than predicted by classical tidal circularization theory ( $\sim 1$ day). Apparently highly dissipative convective envelopes are not necessary for effective circularization!

ZINNECKER: Is it true that the age of the $\alpha$ Persei cluster is only $10^{7}$ years? I thought it was five times that age.

ABT: I suspect that all the ages are incorrect, especially the estimates of the free-fall times. But that doesn't matter at this stage as long as the evolutionary sequence is not altered.

LEINERT: You showed in order to demonstrate the formation of equal-mass binaries, that the total nmber of binaries decreased with time. How seriously can we take this second effect showing in these simulations?

ABT: After a series of binary formations and disruptions, the number of binaries is not conserved. And, the numbers are small. 